NASA-CR-204591

\title{
An inversion of gravity and topography for mantle and crustal structure on Mars
}

\author{
Walter S. Kiefer \\ Lunar and Planetary Institute, Houston, Texas \\ Bruce G. Bills \\ Geodynamics Branch, Goddard Space Flight Center, Greenbelt. Maryland \\ R. Steven Nerem ${ }^{1}$ \\ Space Geodesy Branch, Goddard Space Flight Center, Greenbelt. Maryland
}

\begin{abstract}
Analysis of the gravity and topography of Mars presently provides our primary quantitative constraints on the internal structure of Mars. We present an inversion of the longwavelength (harmonic degree $\leq 10$ ) gravity and topography of Mars for lateral variations of mantle temperature and crustal thickness. Our formulation incorporates both viscous mantle flow (which most prior studies have neglected) and isostatically compensated density anomalies in the crust and lithosphere. Our nominal model has a $150-\mathrm{km}$-thick high-viscosity surface layer over an isoviscous mantle, with a core radius of $1840 \mathrm{~km}$. It predicts lateral temperature variations of up to a few hundred degrees Kelvin relative to the mean mantle temperature, with high temperature under Tharsis and to a lesser extent under Elysium and cool temperatures elsewhere. Surprisingly, the model predicts crustal thinning beneath Tharsis. If correct, this implies that thinning of the crust by mantle shear stresses dominates over thickening of the crust by volcanism. The major impact basins (Hellas, Argyre, Isidis, Chryse, and Utopia) are regions of crustal thinning, as expected. Utopia is also predicted to be a region of hot mantle, which is hard to reconcile with the surface geology. An alternative model for Utopia treats it as a mascon basin. The Utopia gravity anomaly is consistent with the presence of a 1.2 to 1.6 $\mathrm{km}$ thick layer of uncompensated basalt, in good agreement with geologic arguments about the amount of volcanic fill in this area. The mantle thermal structure is the dominant contributor to the observed geoid in our inversion. The mantle also dominates the topography at the longest wavelengths, but shorter wavelengths (harmonic degrees $\geq 4$ ) are dominated by the crustal structure. Because of the uncertainty about the appropriate numerical values for some of the model's input parameters, we have examined the sensitivity of the model results to the planetary structural model (core radius and core and mantle densities), the mantle's viscosity stratification, and the mean crustal thickness. The model results are insensitive to the specific thickness or viscosity contrast of the high-viscosity surface layer and to the mean crustal thickness in the range 25 to $100 \mathrm{~km}$. Models with a large core radius or with an upper mantle low-viscosity zone require implausibly large lateral variations in mantle temperature.
\end{abstract}

\section{Introduction}

In the absence of a lander network providing seismic and heat flow data, gravity and topography are our primary quantitative constraints on the internal structure of Mars. In this work, we present an inversion of Mars gravity and topography for mantle temperature anomalies and crustal thickness varia-

'Now at Department of Aerospace Engineering and Engineering Mechanics, University of Texas at Austin.

Copyright 1996 by the American Geophysical Union.

Paper number 95JE03699.

0148-0227/96/95JE-03699\$09.00 tions. We consider spherical harmonics up to degree 10 , corresponding to wavelengths longer than $2100 \mathrm{~km}$. Accordingly, our analysis emphasizes the major landforms on Mars: Tharsis, Elysium, Valles Marineris, and the large impact basins.

Our model incorporates the effects of viscous mantle flow, something that has not been included in previous inversions of the Martian gravity field. Our approach is generally similar to the Venus model of Herrick and Phillips [1992], although our model differs from theirs in some details. We begin by describing the spherical harmonic data sets used in our inversion, along with a brief consideration of some of the statistical properties of the harmonic models. We then describe the details of our model's formalism. Next, we present results for a series of models, allowing for a plausible range of values for the core radius, mantle viscosity model, and mean crustal 
thickness. We conclude with a brief consideration of the relationship between the stress field predicted by our model and the observed tectonics of the Tharsis region.

\section{Data Sets}

The gravity field of Mars has been estimated using Doppler tracking of the Mariner 9 and Viking 1 and 2 spacecrafts. In this work, we use the spherical harmonic degree 50 model, GMM1, of Smith et al. [1993]. The gravitational potential, $U$, for this field is

$$
\begin{aligned}
U(\theta, \phi, r)= & \frac{\mathrm{GM}}{r}\left(1+\sum_{l=2}^{50} \sum_{m=0}^{\prime}\left(\frac{\mathrm{R}}{r}\right)^{\prime} \mathrm{P}_{l m}(\cos \theta)\right. \\
& \left.\mathrm{x}\left(C_{l m} \cos m \phi+S_{l m} \sin m \phi\right)\right)
\end{aligned}
$$

where $P_{l m}(\cos \theta)$ are normalized associated Legendre polynomials of degree $l$ and order $m, \theta$ is the colatitude, $\phi$ is the longitude, and $r$ is the radius at which the field is evaluated. $C_{l m}$ and $S_{l m}$ are the harmonic coefficients, $G M=42828.28 \mathrm{~km}^{3} \mathrm{~s}^{-2}$ and $\mathrm{R}=3394.2 \mathrm{~km}$ [Smith et al., 1993]. Because of the highly elliptical orbits and various inclinations of the Mariner 9 and Viking Orbiters, the actual resolution of the gravity field varies considerably with latitude and to a lesser extent with longitude. The resulting field has a formal half-wavelength horizontal resolution of $215 \mathrm{~km}$, although in practice, the field only approaches this resolution where low-altitude spacecraft tracking exists to constrain the field. The region of low-altitude coverage extends roughly from $50^{\prime \prime} \mathrm{N}$ to $40^{\prime \prime} \mathrm{S}$ latitude, with the effective resolution of the gravity field being considerably degraded as one approaches the poles. Formal error estimates for the free-air gravity range from about $50 \mathrm{mGal}$ in the equatorial zone to in excess of $80 \mathrm{mGal}$ at the South Pole [Smith et al., 1993]. Another spherical harmonic degree 50 model of the Mars gravity field, Mars50c, has recently been developed [Konopliv and Sjogren, 1995]. GMM1 and Mars50c differ significantly from one another in their high-degree harmonics, but are quite similar in their low-degree harmonics. Because the emphasis in this paper is on the low-degree harmonics, we consider only GMM1; our inversion results are essentially unchanged if Mars $50 \mathrm{c}$ is used instead. The free-air gravity anomaly is $\delta g=-\partial U / \partial r$.

We also use a spherical harmonic degree 50 expansion of the topography of Mars [Bills and Nerem, 1995], of the form

$$
\begin{aligned}
H(\theta, \phi)= & \mathrm{R}\left(1+\sum_{l=1}^{5 l} \sum_{m=0}^{1} \mathrm{P}_{l m}(\cos \theta)\right. \\
& \left.\times\left(A_{l m} \cos m \phi+B_{l m} \sin m \phi\right)\right)
\end{aligned}
$$

where $H$ is the topography at a point and $A_{l m}$ and $B_{l m}$ are the harmonic coefficients. The topography harmonics were derived from a digital version of the current Mars topography model [U.S. Geological Survey (USGS), 1989]. The estimated vertical uncertainty in the input topography model varies from $1 \mathrm{~km}$ near the equator to more than $2 \mathrm{~km}$ near the poles [USGS, 1989]. The USGS topography model is referenced to a Mariner 9 era degree 4 equipotential surface [Jordan and Lorell. 1975]. In contrast, the topography maps and harmonics in this paper are referenced to the degree 50 equipotential surface of Smith et al. [1993]. An additional difference between the
USGS topography and the spherical harmonic model is the zero reference elevation. The USGS topography model is referenced to zero elevation at the 6.1 -mbar pressure level, and in this reference frame the topography has a globally averaged elevation of nearly $2 \mathrm{~km}$. In contrast, in the spherical harmonic model the zero elevation is set by the mean radius $\mathrm{R}$ and any map based on the spherical harmonics will produce a surface with a globally averaged elevation of zero, regardless of the range of harmonic degrees used in the expansion. Thus numerical values from our topographic maps can be directly compared with the values on the USGS maps only if this offset is accounted for. We will sometimes use the shorthand notation $U_{l m}$ to refer collectively to a pair of coefficients $C_{l m}$ and $S_{l m}$ and the notation $H_{l m}$ to refer collectively to a pair of coefficients $A_{i m}$ and $B_{l m}$ at a particular value of $l$ and $m$.

\section{Statistical Analysis of Harmonic Models}

In this section, we briefly discuss some of the statistical properties of the Martian gravity and topography harmonic models. We consider only the long-wavelength portions of these fields, emphasizing issues that are relevant to the inversion developed in the next section. Analysis of the full harmonic degree 50 fields was presented by Bills and Nerem [1995]. Comparisons of Mars with the gravity and topography of Earth, Venus, and the Moon have been discussed by Esposi. to et al. [1992] and Balmino [1993].

The circles in Figure 1 show the gravitational power (the sum of the squares of the coefficients) as a function of harmonic degree. The triangles in Figure 1 show the topography power, expressed in terms of the gravitational potential due to uncompensated topography [Bills et al., 1987, equation (50)]. For degrees 3 to 10 , the gravity power is typically a factor of

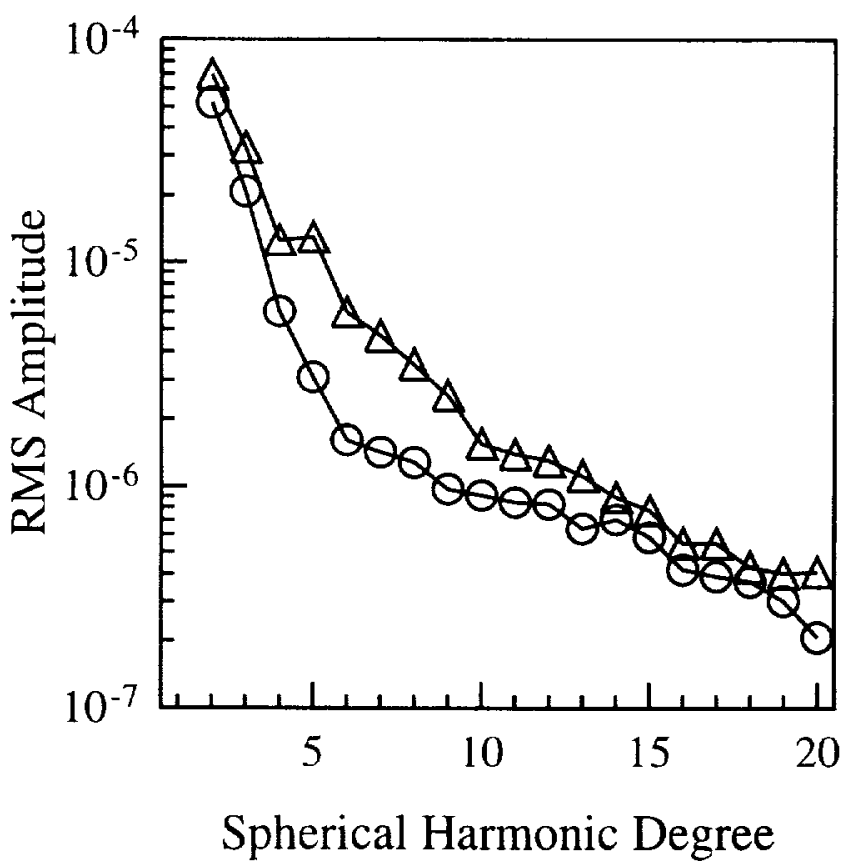

Figure 1. Root-mean-square amplitude of the gravitational potential of Mars (circles) and of the gravitational potential due to uncompensated topography (triangles) as a function of spherical harmonic degree. 
about 2 to 3 less than the uncompensated topography power, implying that the long-wavelength topography of Mars is compensated to a considerable degree. Between harmonic degrees 14 and 19 , the gravity power is always between $71 \%$ and $87 \%$ of the uncompensated topography power, implying that topography in this wave band is largely uncompensated. Maps of the geoid anomaly, free-air gravity anomaly, and topography in the degree 14 to 19 wave band are dominated by high-amplitude positive anomalies centered on Olympus Mons, Arsia Mons, Ascraeus Mons, Alba Patera, and Elysium Mons. These volcanos are all partially supported by elastic flexure [Comer et al., 1985; Zuber et al., 1993; Kiefer et al., 1995].

A more quantitative way to analyze the relationship between gravity and topography is to consider a linear relationship of the form

$$
U_{l m}=F_{1} H_{l m}+I_{l m}
$$

Here, $F_{l}$ is the admittance and $I_{l m}$ are the coefficients for the part of the gravitational potential which is uncorrelated with the topography. The admittance may be estimated using standard least squares methods [e.g., Bills et al., 1987, appendix]. This is shown with one standard deviation uncertainties in Figure 2. For topography which is Airy compensated at a depth $D$ below the surface, the admittance is given by [e,g., Bills et al., 1987]

$$
F_{l}=\frac{3 \rho_{\mathrm{c}}}{(2 l+1) \rho_{\mathrm{av}}}\left(1-\left(1-\frac{D}{\mathrm{R}}\right)^{\prime}\right)
$$

where $\rho_{\mathrm{c}}$ is the assumed crustal density $\left(2900 \mathrm{~kg} \mathrm{~m}^{-3}\right)$ and $\rho_{\mathrm{dv}}$ is the average planetary density $\left(3930 \mathrm{~kg} \mathrm{~m}^{-3}\right)$. This is shown in Figure 2 for $D=100 \mathrm{~km}$ (bottom dashed line), $D=200 \mathrm{~km}$ (middle dashed line), and $D=400 \mathrm{~km}$ (top dashed line). Clearly, no single compensation depth can satisfy the observations [e.g., Phillips and Saunders, 1975; Lambeck, 1979]. The degree 2 and 3 admittances require compensation depths of 1400 and $550 \mathrm{~km}$, respectively, which vastly exceeds any reasonable estimate of the crust's thickness and indicates that dynamic support by mantle convection may play an important role. Moreover, the admittance produced by a convecting system can decline much more rapidly as a function of harmonic degree

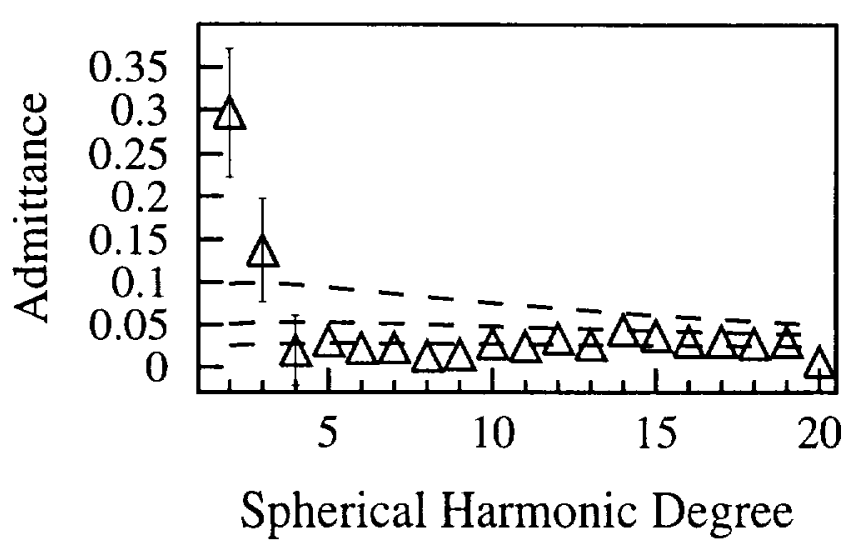

Figure 2. Observed admittances as a function of spherical harmonic degree. One sigma uncertainties are also shown for degrees 2 through 4 . For higher degrees, the uncertainties are smaller than the plotted symbol size. The dashed lines are admittance spectra for Airy compensation at depths of $100 \mathrm{~km}$ (bottom line), $200 \mathrm{~km}$ (middle), and $400 \mathrm{~km}$ (top line).

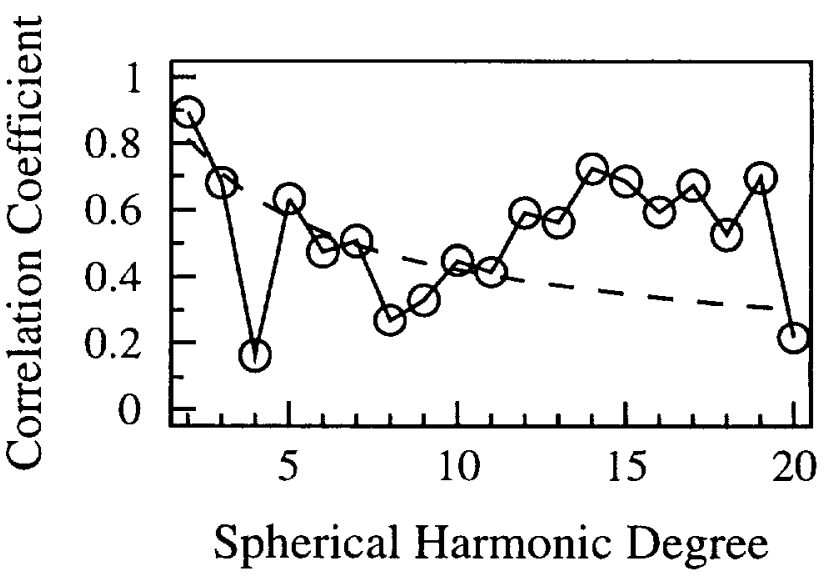

Figure 3. Correlation between gravitational potential and topography on Mars as a function of spherical harmonic degree (circles and solid lines). Dashed line is $95 \%$ statistical confidence limit.

than for either Airy or Pratt compensated layers [Kiefer et al., 1986; Kiefer and Hager, 1991]. The rapid decline in admittance observed for degrees 2,3 , and 4 is therefore also qualitatively consistent with an important role for dynamic support. From degrees 4 to 20, Airy compensation at a depth of $100 \mathrm{~km}$ can account for most of the observations. However, this does not rule out a role for convection at these wavelengths. As shown later in this paper, most of the gravity field and some of the topography may be supported by mantle convection, at least up to the $l=10$ cut off used in our density inversions.

The admittance values in Figure 2 are only meaningful if the linear relationship between gravity and topography (equation (3)) is a good fit to the data. This can be assessed using the linear least squares correlation coefficient. In Figure 3, the circles and solid lines show the correlation between gravitational potential and topography as a function of harmonic degree, while the dashed line shows the $95 \%$ statistical confidence limit. The correlation is statistically significant for degrees $2,5,7$, and 10 to 19 at the $95 \%$ confidence level and for degrees 3 and 6 at the $90 \%$ confidence level. Although the correlation is not statistically significant at a few degrees, enough degrees are well-correlated that we can be sure that the general shape of the admittance spectrum (a rapid decline at low degrees and nearly constant admittance thereafter) is well constrained. The error bars on the admittances in Figure 2 also demonstrate this.

\section{Modeling Procedures}

In this section, we describe our method for inverting gravity and topography for models of the internal mass distribution of Mars. Results from our inversions are presented in the following section. Given the gravitational potential of a planet, one can always solve for density anomalies at any specified depth in the planet which will exactly reproduce the observed potential. However, the resulting mass distribution will not in general be consistent with the planet's topography and common concepts such as isostasy, flexure, or mantle convection. If density anomalies are allowed to exist in two shells at different depths, then it is possible to find a mass distribution which will satisfy both the observed gravitational potential and the topography. The results of such two layer models are non-unique, 
depending on the assumed depths of the density anomalies and on the assumed compensation mechanisms. Nevertheless, by considering a range of plausible models, it is possible to determine the range of density distributions which are consistent with the observed gravity and topography of a planet.

Many prior quantitative analyses of the gravity and topography of Mars have focused on the role of crustal and lithospheric processes. Many of these studies have emphasized the Tharsis region [Sleep and Phillips, 1979, 1985; Banerdt et al., 1982, 1992; Willemann and Turcotte, 1982; Finnerty et al., 1988; Phillips et al., 1990]. Other studies have considered Elysium [Janle and Ropers, 1983], large shield volcanos [Sjogren, 1979; Zuber et al., 1993; Kiefer et al., 1995, Turtle and Melosh, 1995], the crustal dichotomy boundary [Janle, 1983; Phillips, 1988], and impact basins and large craters [Sjogren and Wimberley, 1981; Sjogren and Ritke, 1982; Solomon et al., 1983]. In several of the Tharsis studies, the possible contribution of mantle convection to the gravity and topography was explicitly rejected, although none of the studies cited above actually attempted to model the effects of mantle convection. However, the prolonged history of volcanism in the Tharsis and Elysium regions [Plescia and Saunders, 1979; Scott and Tanaka, 1980; Tanaka and Scott, 1987; Plescia, 1990] can be readily explained by adiabatic decompression of upwelling material. This implies that mantle convection probably has been an important process, at least in these two areas of Mars, perhaps for much of the history of the planet.

The density anomalies associated with this convection will contribute to the gravity and topography. If the elastic lithosphere is very thick, as is true for some of the models cited above, then there may be little dynamic topography produced by the convective flow. Nevertheless, the density anomalies within the convecting mantle will contribute to the gravitational potential; this effect has not been incorporated in any of the models cited above. At most, these models allowed for static, Pratt-compensated density anomalies at depths of up to a few hundred kilometers. However, at the lowest harmonic degrees, density anomalies below the middle of the mantle may still contribute significantly to the gravitational potential [Richards and Hager, 1984]. In addition, the gravity-topography admittance for convecting systems is generally not the same as for a Pratt-compensated layer [Kiefer et al., 1986: Kiefer and Hager. 1991]. Existing models which neglect mantle convection therefore should not be considered as complete or definitive models for the gravity and topography of Mars, but rather as explorations of one type of end-member model.

In this same spirit of exploring end-member models, we consider here another plausible but previously unexplored type of end-member model, in which a combination of mantle convection and isostatically compensated crustal structure exactly satisfies the long-wavelength gravity and topography. For reasons explained in greater detail below, we do not include the effects of elastic flexure of the lithosphere in this study. In reality, it is likely that convection, isostasy, and flexure all play roles in producing the gravity and topography of Mars, and ultimately a model which simultaneously incorporates all three processes will be necessary. However, such a model will of necessity be fairly complex, and it is useful to first understand how each of the end-member mechanisms may contribute separately to the overall picture. The models presented here describe one such end-member. In the longer term, acquisition of improved gravity and topography data sets [Smith et al.,
1990; Zuber et al., 1992] and of seismic and heat flow data from the surface may be necessary to fully resolve the relative roles of isostasy, flexure, and convection. In addition to the inversions presented here, several studies have developed finite element simulations of mantle convection on Mars [Kiefer and Hager, 1989; Schuberf et al., 1990]. The finite element models require fewer approximations but do not exactly reproduce the observed gravity and topography. Our inversions require a greater degree of parameterization but do produce density models which exactly reproduce both the observed gravity and topography. Moreover, the inversions are computationally much more efficient, allowing us to rapidly explore the effects of varying model parameters.

\section{Inversion Method}

Recently, several two-layer models for the gravity and topography of Venus have been presented in which the deeper density layer drives viscous mantle flow and the upper density layer represents crustal structure [Bills and Fischer, 1992; Grimm and Phillips, 1992; Herrick and Phillips, 1992]. The models described here also combine mantle flow and crustal structure. Our approach most closely resembles the global Venus model of Herrick and Phillips [1992], although our implementation differs in some details. We split the observed gravity and topography into two contributions:

$$
\begin{aligned}
& U_{m}^{d}+U_{l m}^{\prime}=U_{l m} \\
& H_{l m}^{d}+H_{l m}^{i}=H_{l m} .
\end{aligned}
$$

Here, $U_{l m}$ and $H_{l m}$ are the observed gravitational potential and topography at degree $l$ and order $m$; recall that this notation implicitly refers to both the sine and cosine terms at a given $l$ and $m$. The terms with superscript $d$ are contributions to the gravity and topography from the deep density anomaly shell. The terms with superscript $s$ are contributions to the gravity and topography from the shallow density anomaly shell. Because we seek to derive information about variations in mantle density and crustal thickness, equations (5)-(7) are all expressed in dimensional terms. Thus the Smith et al. [1993] gravity harmonics are multiplied by $\mathrm{GM} / \mathrm{R}$ prior to being inserted for $U_{m}$ on the right side of equation (5a). Similarly, the Bills and Nerem [1995] topography harmonics are multiplied by $R$ before being inserted for $H_{l m}$ on the right side of equation (5b).

We assume that density anomalies in the deep layer represent mantle temperature variations associated with convective flow. This flow deforms the surface, producing topography. The gravitational potential is the sum of contributions from the mantle mass anomalies and from mass anomalies associated with flow-induced topography at the surface and at the base of the mantle (all three contributions are summed together in the weighting function $X_{l}$ in equation (6a)). The gravitational potential and topography produced by the convective flow can be written as

$$
\begin{gathered}
U_{l m}^{d \prime}=\frac{4 \pi \mathrm{GR}}{2 l+1} \int_{R_{1}}^{R_{2}} X_{l}(r) \delta \rho_{l m}^{d}(r) d r \\
H_{l m}^{d}=\frac{1}{\rho_{\mathrm{sur}}} \int_{R_{1}}^{R_{2}} T_{l}(r) \delta \rho_{l m}^{d}(r) d r
\end{gathered}
$$

In equations (6a) and (6b), G is the gravitational constant, $R$ is 
the planetary radius, $R_{1}$ and $R_{2}$ are the radii of the top and bottom of the shell in which density anomalies are located, and $\rho_{\text {sur }}$ is the density of the uplifted material at the surface. Both the surface of density contrast $\rho_{c}$ and the crust-mantle interface of density contrast $\left(\rho_{m}-\rho_{c}\right)$ are uplifted. Both interfaces contribute to the uplifted mass anomaly, so the sum of the two density contrasts, the mantle density $\rho_{m}$, is used as the value for $\rho_{\text {sur }}$. The functions $X_{l}(r)$ and $T_{I}(r)$ are weighting functions, which are derived by solving the continuity and conservation of momentum equations for the viscous flow problem. We assume a depth-dependent, Newtonian rheology and therefore solve for $X_{I}(r)$ and $T_{i}(r)$ using a propagator matrix technique [Richarls and Hager, 1984]. The propagator matrix formalism allows viscosity to vary with depth but not laterally. In the absence of lateral viscosity variations, $X_{l}$ and $T_{l}$ are independent of spherical harmonic order $m$. The values of $X_{I}$ and $T_{1}$ are functions of the assumed boundary conditions and of the depth-dependence of the viscosity. We assume a no-slip (zero horizontal velocity) top boundary and a free-slip (zero shear stress) boundary at the base of the mantle. The assumed mantle viscosity model is discussed in greater detail below.

Recent two-layer inversions of Venus [Bills and Fischer, 1992; Grimm and Phillips, 1992; Herrick and Phillips, 1992] have all assumed loading at a specified depth in the mantle, in essence making $\delta \rho_{m m}^{d}(r)$ a delta function. Herrick and Phillips did consider various choices of loading depth, but each of their model inversions used a single fixed loading depth. In contrast, we believe it is important to allow loading of the mantle to occur over a range of depths in each inversion. This is because the various harmonic degrees have different depth sensitivities, and because for any given degree, the functions $X_{F}(r)$ and $T_{R}(r)$ have different depth dependences [Richards and Hager, 1984]. We do not have sufficient constraints to independently determine $\delta \rho$ at more than two depths, but we can specify an a priori functional form for $\delta \rho(r)$ and simply solve for a scaling constant. We follow Kiefer et al. [1986] in assuming that the loading is independent of depth in the mantle, that is $\delta \rho(r)=\delta \rho$. This is a reasonable first approximation to some types of convective structures, such as mantle plumes. However, it is likely that there are vertical variations in the mantle thermal structure of Mars, so our results are best considered as a vertical average. Although a more detailed model for $\delta \rho(r)$ would be desirable, we think that the simplified form used here is preferable to ignoring mantle flow altogether, as has been done in all prior quantitative studies of Martian gravity and topography.

Jarvis and Peltier [1986] showed that when the temperature distributions at various depths in a convecting layer are expanded in the wavenumber domain, the thermal boundary layers at the top and bottom of the system have different spectra from the remainder of the system. This implies that the simple approximation that $\delta \rho(r)$ is independent of depth can not be extended to the boundary layers. For this reason, we do not incorporate the upper thermal boundary layer into the integration of equations (6a) and (6b). Instead, we treat the upper thermal boundary layer as part of our shallow density layer and set the integration limit $R_{2}$ to be the average base of the upper thermal boundary layer. The Earth's thermal boundary layer reaches an asymptotic value of $95 \mathrm{~km}$ [Stein and Stein, 1992], with an average thickness of roughly two-thirds of this, or about $60 \mathrm{~km}$. Scaling the heat flux by planetary mass and inversely by surface area suggests that Mars has a heat flux that is about $40 \%$ of Earth's heat flux. The upper boundary layer thickness scales inversely with heat flux, so we adopt $150 \mathrm{~km}$ as the value for $R_{2}$. Strictly speaking, we should also handle the lower thermal boundary layer density model separately from the overlying mantle. However, because both $X_{/}(r)$ and $T_{/}(r)$ go to zero at the base of the convecting layer, the lower thermal boundary layer makes a negligible contribution to the integrals in equations (6a) and (6b). Thus the details of how the lower thermal boundary layer is included in $\delta \rho(r)$ are unimportant.

Our shallow density anomaly layer includes both density variations due to lateral variations in the structure of the upper thermal boundary layer and density variations due to variations in crustal thickness. Density anomalies in the thermal boundary layer create topography by viscous flow, and their gravitational and topographic effects can therefore be treated by the formalism in equations (6a) and (6b). We noted earlier that Pratt compensation is not a good analog for a complete convecting system. However, if one considers just the upper thermal boundary layer of a convecting system, where the loading depth is small in comparison with the horizontal wavelength, then the viscous flow calculation gives results which are essentially the same as for the case of Pratt compensation. In other words, within the upper thermal boundary layer, the function $T_{l}$ is close to its limiting value of -1.0 . In this work, we also assume that variations in crustal thickness are compensated by Airy isostasy. For wavelengths that are much longer than the vertical thickness of the isostatic compensation layer, Airy and Pratt compensation are indistinguishable on the basis of their gravity signatures. For this reason, it is necessary to consider both boundary layer thermal structures and crustal thickness variations as part of the shallow layer. This view contrasts with recent Venus inversions cited earlier, where it was assumed that the effects of the crust and mantle could be separated into different density layers.

Although the foregoing discussion shows that both the upper thermal boundary layer and the crust may contribute to our shallow density layer, it is not possible to specify a priori the relative importance of these two sources. Indeed, the relative strength of the two sources may be different for each term in the harmonic expansion. For the sake of formulating a mathematically well-posed problem, we therefore formulate our inversion assuming that the shallow layer is due solely to crustal thickness variations. However, when interpreting our results, we must remember that boundary layer temperature variations are also part of the shallow layer. When the shallow layer is formulated in terms of crustal thickness variations, it is convenient to let the topography supported by the shallow layer be the independent variable. Thus we may write

$$
\begin{aligned}
& U_{l m}^{*}=\frac{4 \pi \mathrm{GR}}{2 l+1} \rho_{c} H_{l m}^{s}\left(1-\left(1-\frac{D}{\mathrm{R}}\right)^{\prime}\right) \\
& H_{m}^{s}=H_{i m}^{*}
\end{aligned}
$$

Equation (7a) is the relationship between gravitational potential and topography for a crust of density $\rho_{c}$, assuming that the topography is Airy compensated at a depth $D$ below the surface of the planet. The admittance defined in equation (4) is essentially a non-dimensional version of equation (7a). Equation (7b) simply emphasizes that topography is the independent variable for this layer of the inversion. By substituting equations (6a), (6b), (7a), and (7b) into equations (5a) and (5b), we end up with a linear system of two equations in two unknowns, 
a) Mars Topography: degree 2-10

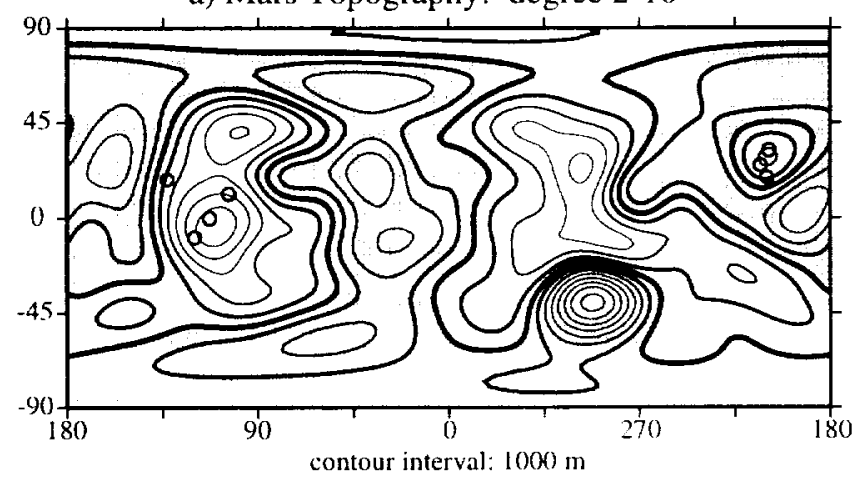

b) Mars Gravity Anomalies: degree 2-10

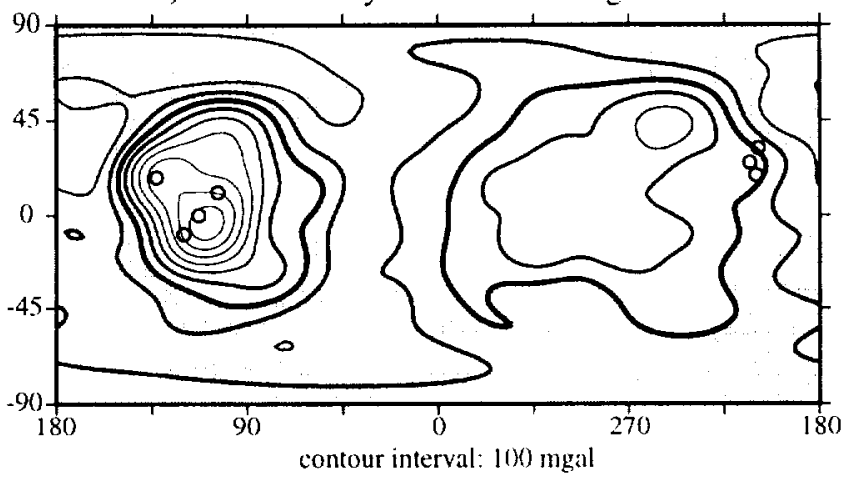

Figure 4. (a) Observed topography of Mars, filtered to include only harmonic degrees 2 to 10 . The contour interval is $1 \mathrm{~km}$, with lows shaded. (b) Observed free-air gravity anomalies of Mars for harmonic degrees 2 to 10 . The contour interval is 100 mGal, with lows shaded. The small circles in these and the other maps represent eight large shield volcanos: Arsia Mons, Ascraeus Mons, Olympus Mons, Pavonis Mons and Alba Patera in Tharsis and Albor Tholus, Elysium Mons, and Hecate Tholus in Elysium.

which is easily inverted for $\delta \rho_{m}^{d}$ and $H_{i m}$. This process is carried out separately for each term in the harmonic expansion.

Because this is an exploratory, global-scale study, we have restricted our inversions to harmonic degrees 2 to 10 , corresponding to a half-wavelength resolution of $1065 \mathrm{~km}$. The observed gravity and topography of Mars filtered to this resolution are shown in Figure 4. Higher resolution studies of some features on Mars are certainly worth pursuing, but are probably best handled in the context of regional rather than global models. Given the resolution of our global model, we emphasize the interpretation of large-scale geologic provinces: major impact basins, the crustal dichotomy, Tharsis, and Elysium.

In this work, we do not consider the effects of elastic lithospheric flexure. This approximation is justified for several reasons. First, the effects of flexure are most important at short wavelengths, whereas we are considering the longest wavelengths on the planet. Second, the specific nature of some of the features of interest here suggests that flexure is unimportant. For example, the crustal dichotomy clearly formed very early in the history of Mars. Theoretically, the mantle heat flow should have been high at that time, and the elastic lithosphere should have been quite thin. Observationally, if flexure were important in supporting the crustal dichotomy, then the 3 to $4 \mathrm{~km}$ of relief across this boundary would be accompanied by a free-air gravity anomaly of about $400 \mathrm{mGal}$. The absence of such a pronounced gravity anomaly along most of the dichotomy boundary indicates that flexure is unimportant in supporting the dichotomy, at least at the wavelengths relevant to this study. The major impact basins also formed early in Martian history. Flexure should therefore be unimportant for the main basin topography, both because the heat flow was high and because impact-induced fracturing further reduced the lithosphere's strength. The long-wavelength gravity anomaly of only $-60 \mathrm{mGals}$ at the $7-\mathrm{km}$-deep Hellas basin is consistent with a lack of flexural support for basin topography. However. as the lithosphere cooled and thickened with time, it might provide some flexural support for later loads emplaced within the basins. We discuss this below in the context of the Utopia basin mascon.

Third, even if elastic flexure were at one time capable of supporting substantial vertical loads, viscous relaxation of elastic stresses will decrease the support of such loads as time progresses. The general trend of planetary thermal evolution is cooling of the interior and thickening of the elastic lithosphere with time. We noted earlier that the prolonged volcanic history at Tharsis and Elysium implies that convective upwelling has probably occurred in these regions for most of the last 4 billion years. In the first billion years of Martian history, the lithosphere must have been quite thin [Schubert and Spohn, 1990] and incapable of resisting convective uplift of the surface. Later, as the planet gradually cooled and the Rayleigh number declined, the convective stresses and hence surface uplift gradually increased [W.S. Kiefer, manuscript in preparation, 1996]. The timescale for this cooling is about $10^{9}$ years [Schubert and Spolin, 1990]. Although the elastic lithosphere tends to resist this increasing convective uplift, the very slow timescale allows viscous relaxation to relieve most or all of the resisting elastic stresses in the lithosphere. Accordingly, surface uplift due to mantle convection is likely to remain in quasi-equilibrium with the underlying thermal structure. Because viscous relaxation occurs most rapidly at long wavelengths [e.g., Solomon et al., 1982], there is no inconsistency between this argument and the observation that elastic flexure contributes to the support of shorter-wavelength features such as shield volcanos [Comer et al., 1985; Zuber et al., 1993; Kiefer et al., 1995]. The hehavior of the gravity power spectrum (Figure 1) noted earlier is in fact quite consistent with little or no flexural support of topography at the lowest harmonic degrees and greater flexural support at high harmonics.

\section{Structural and Viscosity Models}

An important consideration in performing this inversion is the internal structure of Mars, particularly the thickness of the mantle. At present, the only geophysical constraints on the core and mantle structure of Mars come from the mean density and the moment of inertia. The mean density is well constrained, but the moment of inertia is uncertain, with estimates ranging bewween dimensionless values of 0.345 [Bills, 1989] and 0.365 [Katala, 1979]. This uncertainty allows a broad range of possible internal structures. Even if the moment of inertia is specified exactly, uncertainty in the internal structure remains. This is because a two-layer (mantle and core) model requires specification of the core radius, the core density, and the mantle density, but only two constraints (density and mo- 
ment of inertia) are available. We consider a reference structural model with a dimensionless moment of inertia of 0.355 , midway between the values proposed by Kaula [1979] and Bills [1989]. This value of the moment of inertia was also recently advocated on geochemical grounds by Longhi et al. [1992]. Because the two-layer model is underconstrained, we choose to specify that the core's radius is $1840 \mathrm{~km}$, or $54 \%$ of the planetary radius, which is the same fraction of the planetary radius that the Earth's core occupies. The core and mantle densities for this model are $7250 \mathrm{~kg} \mathrm{~m}^{-3}$ and $3300 \mathrm{~kg} \mathrm{~m}^{-3}$. We consider only an average density for the entire mantle and neglect the small $(-6 \%)$ density change across the olivine-spinel phase transition. This has only a slight effect on the calculation of how the gravitational acceleration varies with depth and is unimportant relative to the other uncertainties in the model. For this model, the hydrostatic contributions to the gravitational potential are $-7.585 \times 10^{-4}$ for $C_{20}$ and $2.7 \times 10^{-6}$ for $C_{40}$ [Sleep and Phillips, 1985, Table B21. Hydrostatic contributions to the higher degree zonals are negligible.

Another important consideration in this modeling is how the mantle's viscosity varies with depth. We consider a reference viscosity model that includes a high-viscosity near-surface layer (simulating the cold, high-viscosity thermal boundary layer) over an isoviscous mantle. The surface layer is $150 \mathrm{~km}$ thick and is 100 times the viscosity of the underlying mantle. The thickness of this layer is based on the heat flow scaling arguments outlined above. Finite element simulations have shown that once a high-viscosity lid is included in a convection model, an asymptotic state is reached such that increasing the thickness or viscosity contrast of the high-viscosity layer has a negligible effect on the geoid or topography produced by the convective flow [Kiefer and Hager, 1992]. Increasing the lid's viscosity contrast from 100 to $10^{4}$ in the present inversion has a negligible eftect on the results. Increasing the lid thickness from $150 \mathrm{~km}$ to $250 \mathrm{~km}$ changes the mantle temperature range by less than $5 \%$ and the crustal thickness range by less than $1 \%$. These results indicate that our calculations are not sensitive to the precise choice of high-viscosity lid parameters. The effects of alternative structural and mantle viscosity models are considered later.

\section{Results}

\section{Reference Model}

Figure 5 shows the mantle temperature anomalies inferred from the inversion of the reference model. The inversion actually produces estimates of mantle density anomalies, which are converted to temperature anomalies using the thermal expansion relationship

$$
\delta \rho=-\rho_{\mathrm{m}} \alpha \delta T \text {. }
$$

Here, $\delta \rho$ and $\delta T$ are the density and temperature anomalies, $\rho_{m}$ is the unperturbed mantle density, and $\alpha$ is the thermal expansion coefficient $\left(3 \times 10^{-5} \mathrm{C}^{-1}\right)$. This relationship can be applied separately to each harmonic coefficient. The results in Figure 5 are shown with elevated temperatures in white and cold regions shaded. The total range of temperature anomalies in Figure 5 is $+350 \mathrm{~K}$ to $-210 \mathrm{~K}$, a reasonable range for a convecting mantle. In comparison, an earlier treatment of Tharsis in terms of Pratt compensation would require lateral temperature variations of $2000 \mathrm{~K}$ [Sleep and Phillips, 1979, 1985]. As Sleep and Phillips noted, such a large variation in temperature is implausible, with density differences due to compositional variations being more likely. In contrast with this earlier work, our model requires much smaller temperature variations because these variations extend through the mantle rather than being confined to the upper few hundred kilometers in a thermal boundary layer.

The most prominent feature of Figure 5 is the pronounced region of elevated temperatures beneath Tharsis. There are two temperature maxima beneath Tharsis. One is just southeast of Pavonis Mons, but elongated in the directions of Arsia and Ascraeus Montes. The second is centered on Olympus Mons. The high-temperature region extends northward to include Alba Patera, but the temperature there is not as

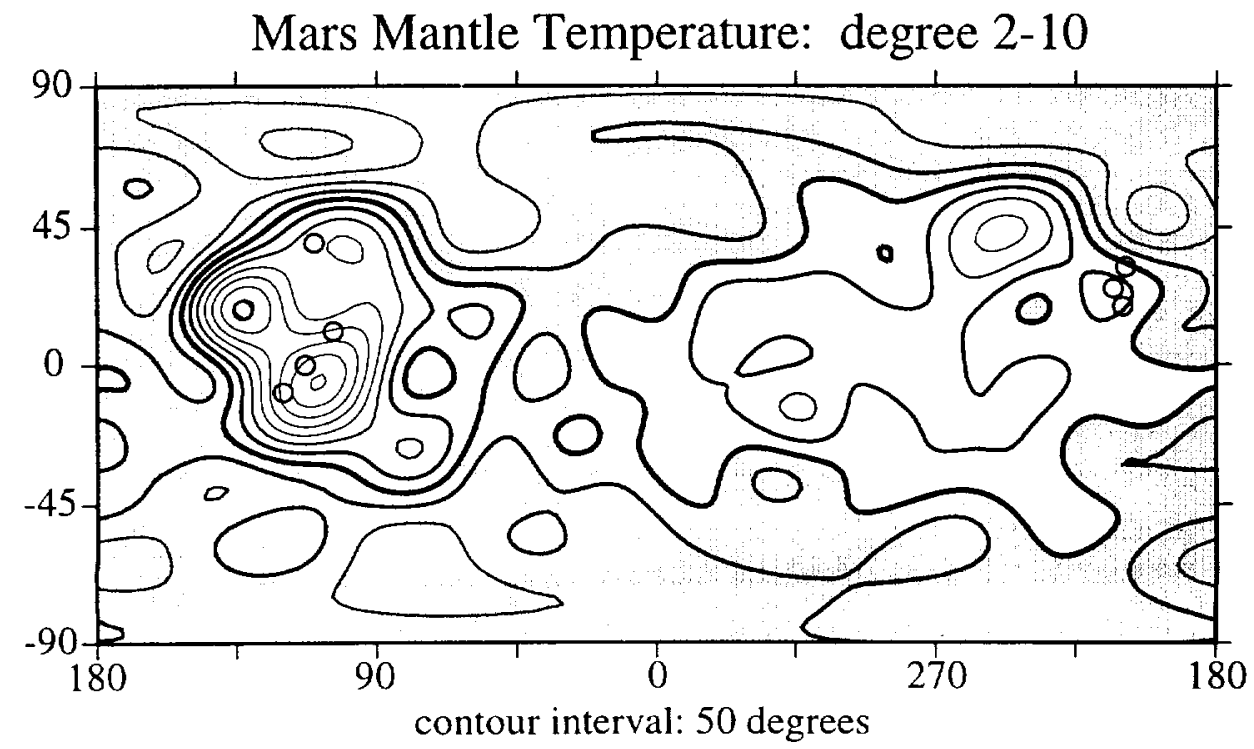

Figure 5. Temperature anomalies in the mantle of Mars for spherical harmonic degrees 2 to 10 for the reference model. The contour interval is $50 \mathrm{~K}$, with negative anomalies shaded. 
high as at either Olympus or Pavonis Montes. The existence of high mantle temperatures in Tharsis is quite consistent with the prolonged history of volcanism in this region. Indeed, the Tharsis region has been volcanically active in geologically recent times [Plescia and Saunders, 1979; Scont and Tanaka, 1980] and may still be active. There is also a hot anomaly in the Elysium region, centered very close to Elysium Mons. The amplitude of this anomaly is smaller than the Tharsis anomaly, consistent with the lower level of volcanic activity in Elysium. The pattern of cold anomalies in Figure 5 does not generally correlate with the surface geology.

The most surprising feature of Figure 5 is the prominent hot anomaly in the Utopia region. This correlates closely with the Utopia impact basin mapped by McGill [1989]. The hot anomaly cannot be a relic of the impact formation process because thermal diffusion will cool impact heating to negligible levels in just a few hundred million years [Bratt et al., 1985], and the Utopia basin is likely to be nearly 4 billion years old. There is evidence for volcanic activity in the early Amazonian in this region, but it is believed due to flows emanating from Elysium Mons rather than being indigenous to the Utopia region (unit Ael3 of Greeley and Guest [1987)). It is possible, however, that earlier Utopia-centered volcanism was covered over by the later Elysium eruptions. McGill [1989] noted the existence of a gravity anomaly which closely correlates with the position of the Utopia basin and suggested that this anomaly is a mascon.

The hot anomaly in Utopia in Figure 5 may be the signature of this mascon. In order to produce a free-air gravity high in a topographic low such as an impact basin, there must be material present which is at least partially uncompensated. Our model requires that shallow loads be isostatically compensated, which means that shallow loads cannot reproduce the observed gravity. In order to produce the observed gravity high, the inversion therefore places a load in the mantle. With the viscosity model used here, a low-density anomaly is needed to produce a positive gravity anomaly [Richards and Hager, 1984]. Thus a flexurally supported, shallow mass excess could show up in our inversion as a high-temperature anomaly in the mantle. In principle, one might also apply this argument to the high-temperature regions in Tharsis, and as noted earlier, several investigators have proposed flexural models for Tharsis. An important difference is that high mantle temperatures in Tharsis are consistent with (and possibly demanded by) the recent volcanic history, an argument that cannot be applied to Utopia. One possible difficulty with the mascon interpretation for Utopia is that proposed mascons in Hellas and Isidis [Sjogren and Wimberley, 1981; Solomon et al., 1983] do not show the same signature in Figure 5 as the Utopia feature. This is not a resolution effect because both Hellas and Isidis show up clearly in the topography map at the same resolution (Figure 4a). However, the gravity anomalies in Hellas and Isidis are smaller in amplitude than in Utopia, so this may not be a serious objection.

The observed free-air gravity anomaly in Utopia is about 150 to $200 \mathrm{mGals}$ relative to its surroundings [Smith et al., 1993]. By measuring the gravity anomaly relative to the surrounding region, we are implicitly assuming that there was no gravity anomaly prior to emplacement of the mascon load. This is a reasonable first-order approximation if the original impact hasin was isostatically compensated, as argued earlier.
The minimum load required to produce the mascon can be derived by assuming that the load is emplaced at the surface (either by volcanism or by sedimentary deposition) and is entirely uncompensated. In this case, the free-air gravity anomaly associated with the load is simply

$$
\delta g(x)=2 \pi \mathrm{G} \rho_{\text {lisud }} \delta h(x),
$$

where $\rho_{\text {load }}$ and $\delta h(x)$ are the density and thickness of the load. This assumes a planar geometry for the load, but spherical geometry will not significantly alter this for a gravity anomaly which is $2000 \mathrm{~km}$ across. Assuming $\rho_{\text {loidd }}=2900 \mathrm{~kg} \mathrm{~m}^{-3}$ (basalt), the load would need to be 1.2 to $1.6 \mathrm{~km}$ thick to produce the observed gravity anomaly. The Elysium flows mapped by Greeley and Guest [1987] in this region could be a major contributor to this load. Sedimentary deposits surround the volcanic flows [Lucchitta et al., 1986] and might also underlie the Elysium volcanic flows, thus contributing to the inferred load thickness. If the load density is less than assumed (e.g., unconsolidated sediments) or the load is partially compensated, then a greater load thickness is necessary to reproduce the observed gravity anomaly. Based on geological relation. ships in Utopia, Frey and Schultz [1990] estimated a minimum volcanic fill of $1 \mathrm{~km}$, with a more likely value of $2 \mathrm{~km}$. This is in good agreement with the estimate derived here, particularly if the load is partially compensated.

Figure 6a shows the shallow layer loads for this inversion. The loads are shown in terms of isostatically supported surface topography, with a range of -7.7 to $+4.8 \mathrm{~km}$. The total variation in crustal thickness (surface relief plus relief on the crust-mantle interface) will be larger by a factor $\rho_{m} /\left(\rho_{m}-\rho_{c}\right)$, where $\rho_{c}$ and $\rho_{m}$ are the crust and mantle densities. Pratt compensated thermal loads in the upper thermal boundary layer can also contribute to this shell. One can convert between Airy and Pratt supported loads using the relationship

$$
\rho_{c} \delta h^{s}=L \rho_{m} \alpha \delta T^{s}
$$

Here, $\delta r^{s}$ is the lopography supported by Airy isostasy and $\delta T^{s}$ is the boundary layer temperature anomaly averaged through a thermal lithosphere of thickness $L$. The superseript $s$ emphasizes that these are the loads from the shallow layer portion of the inversion. Equation (10) allows one to convert from pure Airy compensation (Figure 6a) to pure Pratt compensation; in the more general case, a combination of both Airy and Pratt isostasy may apply at any given point. Crustal thickening is shown in white in Figure 6a and crustal thinning is shaded. As expected, the major impact basins (Hellas, Argyre, Isidis, Utopia, and Chryse) are all regions of thinned crust in Figure 6a. Except where affected by these basins, areas on the south side of the crustal dichotomy boundary are typically areas of thickened crust. Regions near the north pole also show up as thick crust, but this simply reflects a degree 1 component in the topography that is not included in our inversion. The region around Valles Marineris is thickened crust (note that the actual trough system cannot be resolved at spherical harmonic degree 10). This crustal thickening is elongated to the east and west. in the direction of Valles Marineris itself.

Figure 6a also shows considerable crustal thinning in Tharsis. The minimum shallow layer topography in the Tharsis region in Figure $6 \mathrm{a}$ is $-5 \mathrm{~km}$ at Olympus Mons, implying a total crustal thinning of about $41 \mathrm{~km}$ for crust and mantle densities of 2900 and $3300 \mathrm{~kg} \mathrm{~m}^{-3}$. The shallow layer topography in 
a) Mars Shallow Layer Topography: degree 2-10

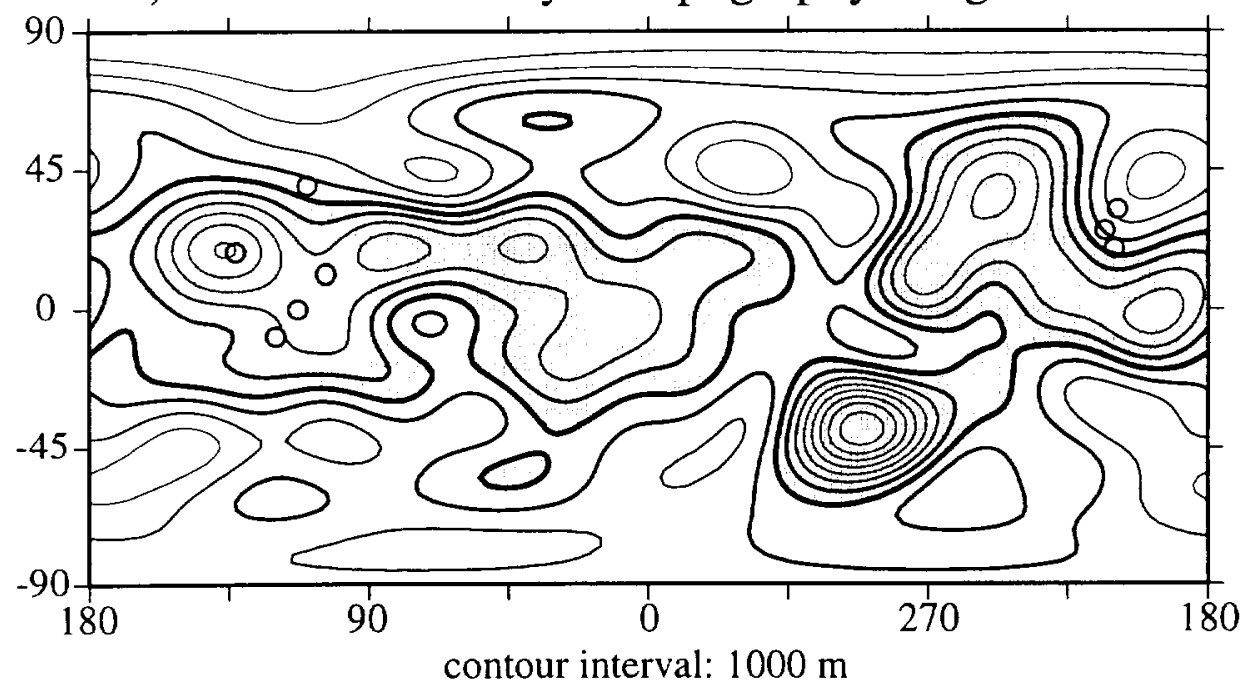

b) Mars Deep Layer Topography: degree 2-10

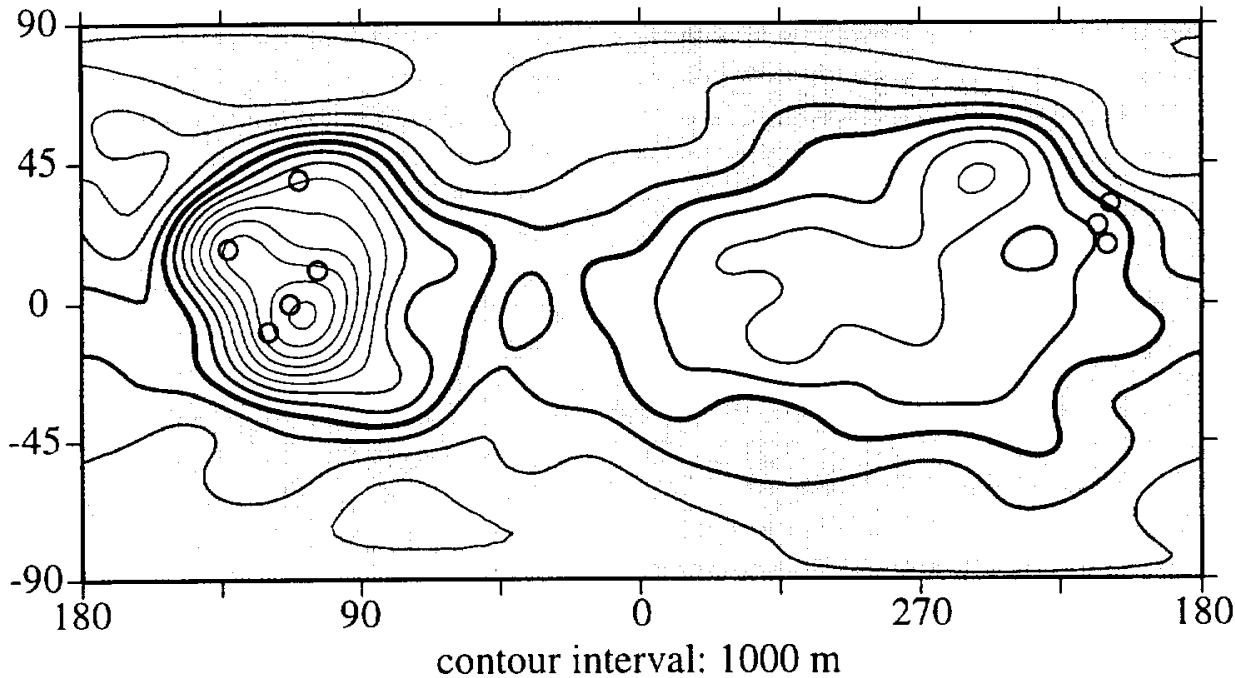

Figure 6. Surface topography produced by the two density anomaly shells for spherical harmonic degrees 2 to 10 for the reference model. The contour interval is $1 \mathrm{~km}$, with negative topography shaded. (a) Topography supported by shallow shell. (b) Topography supported by deep shell.

central Tharsis is only about $-2 \mathrm{~km}$, implying about $16 \mathrm{~km}$ of crustal thinning. Given the hot mantle temperature beneath Tharsis (Figure 5), we would also expect the thermal boundary layer to be hotter than normal. A hot boundary layer affects the gravity and topography in a manner analogous to thickened crust (equation (10)). Thus, if a hot boundary layer is present in Tharsis, the actual amount of crustal thinning must be even larger than shown in Figure 6a in order to compensate for the effects of the boundary layer.

Considering the prolonged history of volcanism in Tharsis, the predicted crustal thinning seems unexpected. The most likely explanation is that mantle shear stresses at the base of the crust are able to thin the crust more rapidly than volcanic processes are able to thicken the crust. The prediction of crustal thinning in Tharsis resembles the model of Sleep and Phil- lips [1979]. Since the work of Sleep and Phillips [1979], crustal thickening in Tharsis has been generally preferred [Solomon and Head, 1982; Finnerty et al., 1988; Phillips et al., 1990]. These models all presume that the crust and low-density residuum produced by magmatic activity remain confined in Tharsis. Our results suggest that this presumption may be incorrect. However, more detailed modeling is clearly necessary to assess the conditions under which basal shearing can thin the crust more rapidly than magma production can thicken it. Such modeling will require the addition of a magma production model to a finite element simulation of the temperature and velocity fields in the mantle.

There are two ways in which the predicted crustal thinning in Tharsis could be reduced. First, if the regional topography in Tharsis is larger than assumed here (a possibility sug. 
gested by recent interpretation of Viking radio occultation observations [Smith and Zuber, 1995]), the actual and model dynamic topography would agree more closely, reducing the need for crustal thinning. Second, the mantle loading model assumes that the density anomalies are uniformly distributed with depth. Because the low-degree geoid weighting functions $\left(X_{1}(r)\right)$ peak in the middle portion of the mantle and the corresponding topography weighting functions $\left(T_{(}(r)\right)$ always peak at the surface [Richards and Hager, 1984], changing the distribution of the density loading in equations (6a) and (6b) will alter our results to some extent. In particular, if there is enhanced loading in the middle of the mantle, the same geoid anomaly could be achieved with a lesser amount of dynamic topographic uplift, which in turn would reduce the required amount of crustal thinning in Tharsis. Such a loading model might arise in time-dependent convection, for example, from an instability which forms in the lower thermal boundary layer and rises toward the surface. Clearly, a range of models can satisfy the observed geoid and topography. Observations from a seismic network [Solomon et al., 1991] ultimately may be required to definitively determine the extent to which crustal thickening or thinning has occurred in Tharsis.

Figure 6a shows that the shallow-layer topography has a small amplitude at the Elysium volcanos, about $1 \mathrm{~km}$ at Elysium Mons. The relatively small shallow-layer topography shown for Elysium could be entirely due to elevated thermal boundary layer temperatures, allowing the possibility that Elysium, like Tharsis, is a region of crustal thinning. However, the amplitude of crustal thinning must be less in Elysium than in Tharsis. Based on the observed topography and gravity (Figure 4), the horizontal scale for convective flow beneath Elysium is smaller than for convective flow beneath Tharsis. Such differences in the horizontal scale of convection have a substantial effect on the geoid and dynamic topography produced by the flow [Kiefer and Hager, 1992], and we speculate that it also may affect the relative balance of crustal thickening or thinning produced by the flow. The maximum shallow-layer topography amplitude in this area is located about $1000 \mathrm{~km}$ to the northeast of Elysium Mons. The amplitude of this maximum $(3 \mathrm{~km})$ is sufficiently large that for plausible values of the boundary layer temperature contrast $\left(\delta T^{s}\right)$, some crustal thickening is required.

Figure $6 \mathrm{~b}$ shows the surface topography supported by the mantle layer. The planform is quite similar to the temperature

Mars Shallow Layer Gravity Anomalies: degree 2-10

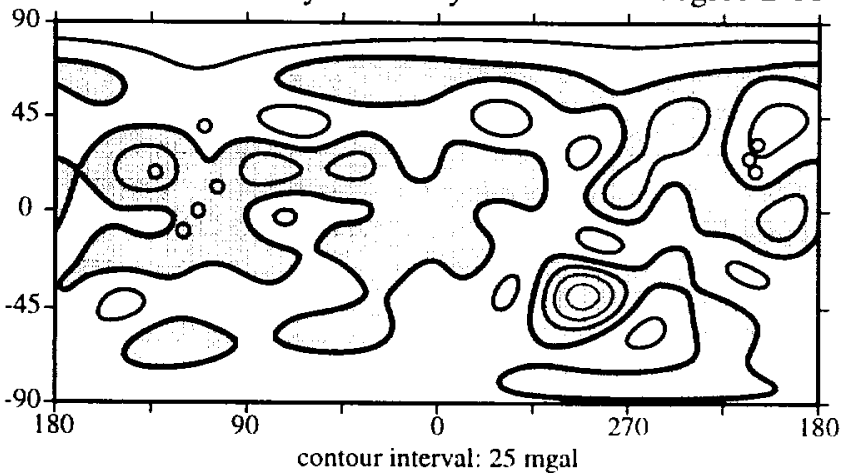

Figure 7. Free-air gravity anomalies produced by the shallow shell for spherical harmonic degrees 2 to 10 of the reference model. The contour interval is $25 \mathrm{mGal}$, and negative anomalies are shaded.

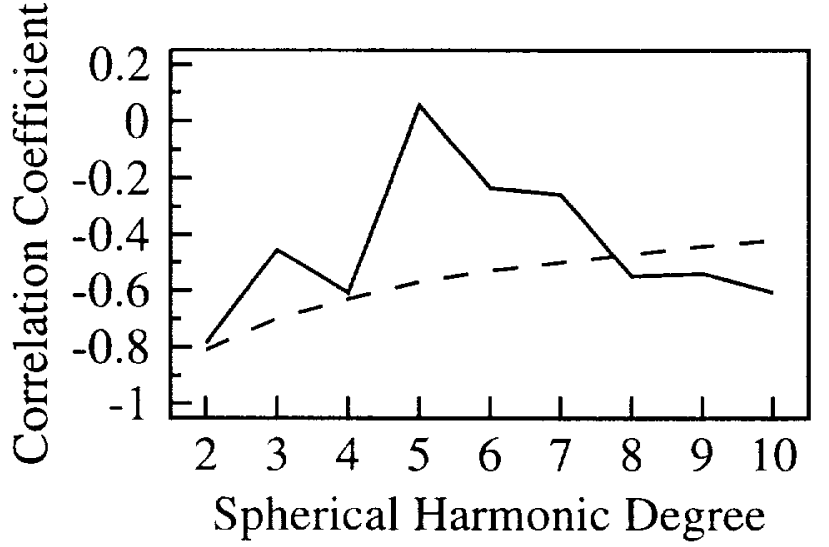

Figure 8. Correlation coefficient as a function of harmonic degree between the topography supported by the shallow density layer and the topography supported by the deep density layer for the reference model. Dashed line is $95 \%$ confidence limit.

anomalies in Figure 5, with pronounced topographic uplift in Tharsis. Comparing Figures $6 a$ and $6 b$, it is clear that the two density shells support comparable amounts of total topographic relief. However, as discussed below, the relative contributions of the two shells to the topography is a strong function of wavelength. In contrast with the topography, the mantle shell is the dominant contributor to the observed gravity. The gravity anomaly due to the deep shell is not shown because it is virtually identical to the observed gravity (Figure 4b). Figure 7 shows the gravity anomaly due to the shallow shell, which has an amplitude of less than $25 \mathrm{mGal}$ in most places, with the notable exception of Hellas, where the shallow-shell anomaly $(-80$ $\mathrm{mGal}$ ) represents most of the total anomaly. The dominance of the deep shell in the gravity field is not surprising because the crustal loads are isostatically compensated at shallow depths, implying small associated gravity anomalies.

Figure 8 shows the correlation between the topography supported by the deep and shallow shells as a function of harmonic degree. The correlation for the gravity produced by the deep and shallow shells is identical. The dashed line is the $95 \%$ statistical confidence limit for each harmonic degree. The correlation between the two shells is negative at all harmonics except degree 5, but only degrees 8,9 , and 10 are statistically significant at the $95 \%$ confidence level. In addition, degrees 2 and 4 are significant at the $90 \%$ level. Recall that the shallowlayer shell may include contributions both from the upper thermal boundary layer and from crustal thickness variations. The thermal structure of the upper boundary layer should be positively correlated with the deep-layer thermal structure. The generally negative correlations shown in Figure 8 therefore are evidence for a significant crustal contribution to the shallow-layer density structure

Figure 9 considers the relative contribution of the two shells to the gravitational potential and the topography as a function of harmonic degree. This is expressed in terms of the fractional power in the gravity field due to the deep shell,

$$
f(l)=\frac{\sum_{m=1}^{\prime}\left(U_{i m}^{\prime \prime}\right)^{2}}{\sum_{m=1}^{\prime}\left(U_{l m}^{d}\right)^{2}+\left(U_{i m}^{s}\right)^{2}} .
$$




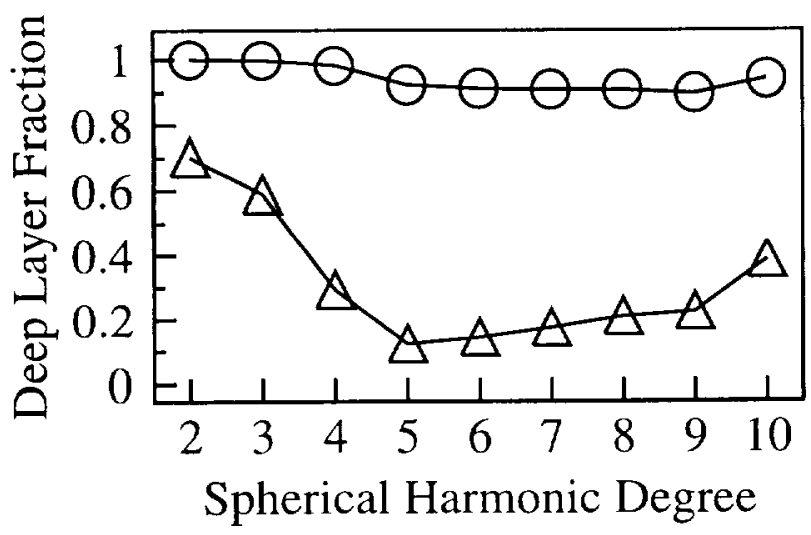

Figure 9. Fractional contribution of deep density layer to the gravitational potential (circles) and the topography (triangles) of the reference model.

Here, the numerator is the power in the deep-shell geoid and the denominator is the power of both the deep- and shallowlayer geoids. The fractional topographic power due to the deep shell can be defined in a similar manner. The circles in Figure 9 are the deep layer fractional power for the geoid, which shows that the deep layer dominates the gravitational potential, with fractional power exceeding $90 \%$ at all wavelengths. On the other hand, the topography results (triangles) show that the lower shell strongly dominates only the low-degree topography, with the shallow shell dominating at $l \geq 4$.

\section{Variation of Model Parameters}

We have examined the effects of varying the planetary structural model, the mantle viscosity model, and the depth of the shallow-layer density anomalies on our model results. The map patterns produced by these various models are generally quite similar to those shown in Figures 5 to 7 , with the results differing primarily in the amplitude of the required density anomalies. For the sake of brevity, we therefore omit the presentation of map results for these models and instead just summarize the amplitude results.

Schubert and Spolm [1990] tabulated structural models of Mars for various choices of moment of inertia and core density. To test the sensitivity of our choice of reference model parameters, we have considered their models with the largest and smallest core radii with our reference viscosity model. Their model with the smallest core has a core radius of 1546 $\mathrm{km}$, a mantle density of $3500 \mathrm{~kg} \mathrm{~m}^{-3}$, and a moment of inertia of 0.365 . The range of mantle temperature anomalies in this model is -150 to $+280 \mathrm{~K}$, somewhat less than for our reference model. Decreasing the core radius below our reference value has a relatively small effect on the required density anomalies. This is because density anomalies below $1500 \mathrm{~km}$ depth in the Mars mantle have little affect on either the geoid or the surface topography. Thus the required range of $\delta T$ in the mantle does not differ greatly in these two models. The difference is due in part to differences in the mantle density in the two models. The gravity and topography which the models must support are sensitive to the density variations in the mantle, but we have converted this to temperature anomalies here. This conversion is inversely proportional to the nominal mantle density (equation (8)), which therefore requires a larger range of $\delta T$ in the refer- ence model, which has a smaller mantle density. Much of the remaining difference is due to differences in the hydrostatic correction to the $C_{21}$ gravity term for the two different structural models. The range of crustal topography in the two models differs by less than 10\%. Recently, Fei et al. [1995] have reported new mineral physics results that allow the core of Mars to have a radius of just $1300 \mathrm{~km}$. Based on the similarity of the two model calculations described here, we do not think that further decreasing the core size will significantly alter our model results.

The model with the largest core in the Schubert and Spoin [1990] tabulation has a core radius of $2468 \mathrm{~km}$, a mantle density of $2785 \mathrm{~kg} \mathrm{~m}^{-3}$, and a moment of inertia of 0.345 . This large core model requires mantle temperature anomalies ranging between -570 and $+850 \mathrm{~K}$, much larger than for the reference model. Because the mantle density in the large core model is $15 \%$ less than in the reference model, the conversion from density anomaly to temperature anomaly (equation (8)) implies larger temperature anomalies in the large core model. Also, the mantle is only $900 \mathrm{~km}$ thick in the large core model and $1550 \mathrm{~km}$ thick in the reference model. A larger temperature contrast is needed to compensate for the reduced depth range of the integration (equations $(6 a)$ and $(6 b)$ ) in the large core model. The Earth's upper mantle temperature is about $1700 \mathrm{~K}$ [Stein and Stein, 1992], and because of the strong temperature-dependence of rheology, if convective flow is significant in the mantle of Mars, then the mean mantle temperature on Mars cannot be more than 100 to $200 \mathrm{~K}$ less than the Earth's temperature. With temperature anomalies of up to $+850 \mathrm{~K}$ relative to the mean mantle temperature, the large core model would require large-scale melting in the mantle, for which there is no evidence in the recent volcanic history of Mars. Accordingly, we must conclude either that such a large core does not exist on Mars or that mantle convection is not a primary contributor to the present-day long-wavelength geoid and topography of Mars.

We have also considered the effect of varying the mantle viscosity structure. The isoviscous mantle used in the reference model is Venus-like [Kiefer and Hager, 1991]. On the other hand, models of the Earth's mantle viscosity structure increase strongly with depth [e.g., Hager and Richards, 1989]. As one test of the effects of a viscosity increase with depth on our results, we have used our reference structural model with a lower mantle that is a factor of 30 more viscous than the upper mantle, with the transition assumed to occur at a depth of 800 $\mathrm{km}$, near the middle of our reference mantle. Increasing the viscosity with depth in the mantle weakens the coupling between convective stresses and the surface. This decreases the amplitude of the dynamic topography produced by the convective flow and also has a large effect on the geoid [Richards and Hager, 1984]. Including a low-viscosity upper mantle layer requires mantle temperature anomalies between -700 and +1300 $\mathrm{K}$. The large positive temperature anomalies required in this model are implausible, as was the case for the model with a large core. This implies either that Mars does not have a strong increase in mantle viscosity with depth or that mantle convection is not the dominant contributor to the present-day geoid and topography on Mars.

The compensation depth in equation (7a) represents a weighted average of the mean crustal thickness and the middle of the upper thermal boundary layer. In the reference model, this was assumed to be $50 \mathrm{~km}$, but we have considered values 
of this parameter between 25 and $100 \mathrm{~km}$. Compensating the shallow-density anomalies at $25 \mathrm{~km}$ represents a situation where the shallow layer is predominantly due to crustal thickness variations in a crust with a small mean thickness. Compensating the density anomalies at $100 \mathrm{~km}$ could represent either a very thick crust or a situation in which the shallow-layer density anomalies primarily represent boundary layer temperature variations. The inversion results are relatively insensitive to the particular value of $D$. Mantle temperature anomalies change by only $10 \%$ and the crustal topography changes by $20 \%$ over this range of $D$. Increasing the compensation depth of the shallow shell increases the contribution of the shallow shell to the gravity field. However, even for $D=100 \mathrm{~km}$, the deep shell dominates the gravity, with the shallow shell contributing no more than $30 \%$ of the power. The fractional contribution of the two layers to the topography is virtually independent of the assumed value of $D$.

\section{Tectonic Implications}

Another method for testing internal structure models of the sort described in this paper is to compare the surface stresses predicted by the models with the observed orientation of tectonic features. Because tectonic features in old terrains on Mars may be unrelated to the present-day gravity and topography, such comparisons have been confined to the Tharsis and Elysium regions [Banerdt et al., 1982, 1992; Willemann and Turcotte, 1982; Sleep and Phillips, 1985; Hall et al., 1986]. Some of the predicted stress patterns in these studies may be modified by considerations of the effects of time-dependent lithospheric loading [McGovern and Solomon, 1993] and of improved lithospheric failure criteria [Sc/huttz and Zuber, 1994]. Although we have not made a detailed analysis of the horizontal stresses implied by the models in this paper, the following analysis suggests that our models are generally consistent with the state of stress implied by the observed tectonics.

Because Tharsis is approximately axisymmetric [Willemann and Turcotte, 1982], we have examined the surface stresses predicted by several spherical axisymmetric mantle convection simulations [Kiefer, 1993; W.S. Kiefer and L.H. Kellogg, manuscript in preparation, 1996]. Although the details are complicated due to the time-dependent nature of these convection simulations, in general, the predicted stresses imply extensional features (graben) oriented radial to Tharsis near the center of the uplift and compressional features (ridges) that are oriented concentric to Tharsis at larger distances from the center of uplift. This is generally consistent with the pattern of faulting observed in Tharsis. Inclusion of the stresses associated with the predicted crustal thinning in Tharsis in our model will modify the predicted tectonics. However, because the topography produced by our mantle load is up to 4 times as large as the shallow-layer topography in central Tharsis, we expect that the crustal layer will only be a small perturbation on the tectonics predicted using just the mantle flow model. A more detailed treatment, including a discussion of how the timedependent convective stresses maly be related to the temporal history of faulting in Tharsis, will be presented elsewhere [W.S. Kiefer, manuscript in preparation, 1996].

\section{Conclusions}

We have presented a series of inversions of the gravity and topography of Mars for mantle and crustal structure. Our models incorporate the effects of viscous mantle flow (which was neglected in prior models of the long-wavelength gravity field of Mars) and of isostatically compensated density anomalies in the crust and lithosphere. These models predict hot upwellings beneath Tharsis and Elysium, consistent with the long volcanic histories of these areas. Crustal thinning is predicted beneath Tharsis, which if correct, implies that thinning of the crust by mantle shear tractions has dominated over crustal thickening due to volcanism. The large impact basins are all regions of thinned crust, and Utopia contains a mascon whose gravity anomaly is consistent with 1.2 to $1.6 \mathrm{~km}$ of uncompensated basalt, consistent with independent geological estimates of the amount of volcanic fill in this region.

We have tested our model for sensitivity to assumed crustal thickness, mantle viscosity model, and core radius. The general planform of mantle and crustal structure does not depend on the assumed values of these parameters, but the amplitudes of the required temperature and crustal thickness variations do depend on these parameters. Models with a small to intermediate size core and without a low-viscosity zone in the upper mantle require lateral temperature variations of a few hundred degrees Kelvin. Models with a very large core or with an upper mantle low-viscosity layer require unreasonably large lateral variations in mantle temperature and can therefore be rejected. These results are fairly insensitive to the mean crustal thicknesses and to the thickness and viscosity contrast of the near-surface layer. In all of these models, the mantle thermal structure is the dominant contributor to the gravitational potential. The mantle also dominates the topography at the longest wavelengths, but harmonic degrees $\geq 4$ are dominated by the crustal structure. The predicted stress distribution is generally consistent with the observed distribution of extensional and compressional features in the Tharsis region.

Acknowledgments. W.S.K. began this work while on a National Research Council Research Associateship at the Goddard Space Flight Center. Most of this work was performed at the Lunar and Planetary Institute, which is operated by the University Space Research Association under NASA contract NASW4574. We thank Maria Zuber for discussions, Robbie Herrick and Scott Murchie for comments on a draft manuscript, and Bruce Banerdt and an anonymous referee for helpful reviews. LPI Contribution Number 878.

\section{References}

Balmino, G., The spectra of the topography of the Earth, Venus, and Mars, Geophys. Res. Lett., 20, 1063-1066, 1993.

Banerdt, W.B., R.J. Phillips, N.H. Sleep, and R.S. Saunders, Thick shell tectonics on one-plate planets: Applications to Mars, J. Geophys. Res.. 87, 9723-9733, 1982.

Banerdt, W.B., M.P. Golombek, and K.L. Tanaka, Stress and tectonics on Mars, in Mars, edited by H.H. Kieffer, B.M. Jakosky, C.W. Snyder, and M.S. Matthews, pp. 249-297, Univ. of Arizona Press, Tucson, 1992.

Bills, B.G., The moments of inertia of Mars, Geophys. Res. Lett., 16. $385-388,1989$.

Bills, B.G., and M.A. Fischer, A spatial domain Stokes flow model for the gravity and topography of the middle latitudes of Venus, $J$. Geophys. Res. 97, 18,285-18,294, 1992.

Bills, B.G., and R.S. Nerem, A harmonic analysis of Martian topography, J. Ceophys. Res., 100, 26,317-26,326, 1995.

Bills, B.G., W.S. Kiefer, and R.L. Jones, Venus gravity: A harmonic analysis, J. (ieophys. Res., 92, 10,335-10,351, 1987. 
Bratt, S.R., S.C. Solomon, and J.W. Head, The evolution of impact basins: Cooling, subsidence, and thermal stress, J. Geophys Res., 90, 12,415-12,433, 1985.

Comer, R.P., S.C. Solomon, and J.W. Head, Mars: Thickness of the lithosphere from the tectonic response to volcanic loads, Rev Geophys., 23, 61-92, 1985.

Esposito, P.B., W.B. Banerdt, G.F. Lindal, W.L. Sjogren, M.A. Slade, B.G. Bills, D.E. Smith, and G. Balmino, Gravity and topography, in Mars, edited by H.H. Kieffer, B.M. Jakosky, C.W. Snyder, and M.S. Matthews, pp. 209-248, Univ. of Arizona Press, Tucson, 1992.

Fei, Y., C.T. Prewitt, H. Mao, and C.M. Bertka, Structure and density of FeS at high pressure and high temperature and the internal structure of Mars, Science, 268, 1892-1894, 1995.

Finnerty, A.A., R.J. Phillips, and W.B. Banerdt, Igneous processes and closed system evolution of the Tharsis region of Mars, J. Geo. phys. Res., 93, 10,225-10,235, 1988.

Frey, H.V., and R.A. Schultz, Speculations on the origin and evolution of the Utopia-Elysium lowlands of Mars, J. Geophys. Res.. 95, $14,203-14,213,1990$.

Greeley, R., and J.E. Guest, Geologic map of the eastern equatorial region of Mars, U.S. Genl. Survey Misc. Inv. Map 1-1802B. 1987.

Grimm, R.E., and R.J. Phillips, Anatomy of a Venusian hot spot: Geology, gravity, and mantle dynamics of Eistla Regio, J. Geophys. Res., 97, 16,035-16,054, 1992.

Hager, B.H., and M.A. Richards, Long-wavelength variations in Earth's geoid: Physical models and dynamical implications, Phil Trans. R. Soc: London A, 328, 309-327, 1989.

Hall, J.L., S.C. Solomon, and J.W. Head, Elysium region. Mars: Tests of lithospheric loading models for the formation of tectonic features, J. Geophys. Res., 9/, 11,377-11,392, 1986.

Herrick, R.R., and R.J. Phillips, Geolngical correlations with the interior density structure of Venus, J. Geophys. Res., 97, 16,017$16,034,1992$

Janle, P., Bouguer gravity profiles across the highland-lowland escarpinent on Mars, Moon and Planets, 28, 55-67, 1983.

Janle, P., and J. Ropers, Investigation of the isostatic state of the Elysium done on Mars by gravity models, Phys. Earth Planet. Interiors, 32, 132-145, 1983.

Jarvis, G.T., and W.R. Peltier, Lateral heterogeneity in the convecting mantle, J. Geophys. Res., 9I, 435-45I, 1986.

Jordan, J.F., and J. Lorell, Mariner 9: An instrument of dynamical science, Icarus, 25, 146-165, 1975.

Kaula, W.M., The moment of inertia of Mars, Geophys. Res. Letl., 6 , 194-196. 1979.

Kiefer, W.S., Time-dependent geoid anomalies and dynamic topography from high Rayleigh number mantle convection (abstract), Eos. Trans. AGU, 74, (Fall Meeting Sipplement), 79-80, 1993.

Kiefer, W.S., and B.H. Hager, The role of mantle convection in the origin of the Tharsis and Elysium provinces of Mars (abstract), in Early Tectonic and Volcanic Evolution of Mars, LPI Tech. Report 89-04, 48-50, 1989.

Kiefer, W.S., and B.H. Hager, A mantle plume model of the equatorial highlands of Venus, J. Geophys. Res.. 96, 20,947-20,966. 1991.

Kiefer, W.S., and B.H. Hager, Geoid anomalies and dynamic topography from convection in cylindrical geometry: Applications to mantle plumes on Earth and Venus, Geophys. J. Int., 108. $198-214,1992$

Kiefer, W.S., M.A. Richards, B.H. Hager, and B.G. Bills, A dynamic model of Venus's gravity field, Geophys. Res. Lets., 13, 14-17, 1986.

Kiefer, W.S., M.T. Zuber, and K.K. Williams, Modeling gravity anomalies at Martian shield volcanos: A reduced estimate of elastic lithosphere thickness at Olympus Mons (abstract), Lunar Planet. Sci. Comf., 26, 743-744, 1995.

Konopliv, A.S., and W.L. Sjogren. The JPL Mars gravity field Mars50c based upon Viking and Mariner 9 Doppler tracking data, $J P L$ Pub. 95-5, 1995.

Lambeck, K., Comments on the gravity and topography of Mars, $J$. Gerphlys. Res., 84, 6241-6247, 1979.

Longhi, J., E. Knittle, J.R. Holloway, and H. Wanke, The bulk composition, mineralogy and internal structure of Mars, in Mors. edited by H.H. Kieffer, B.M. Jakosky, C.W. Snyder, and M.S Malthews, pp. 184-208, Univ. of Arizona Press, Tucson, 1992.

Lucchitta, B.K., H.M. Ferguson, and C. Summers, Sedimentary deposits in the northern lowland plains, Mars, Proc. Lunar and Planet. Sci. Conf., 17th. J. Geophys. Res., 91 (supplement), E166-E174, 1986.

McGill, G.E., Buried topography of Utopia, Mars: Persistence of a giant impact depression, J. Geophys. Res., 94, 2753-27.59, 1989.

McGovern, P.J., and S.C. Solomon, State of stress, faulting, and eruption characteristics of large volcanoes on Mars, J. Geophys. Res., 98. 23,533-23,579, 1993.

Phillips, R.J.. The geophysical signal of the Martian global dichotomy (abstract), EOs, Trans. $A G U, 69,389,1988$.

Phillips, R.J., and R.S. Saunders, The isostatic state of Martian topography, J. Geophys. Res., 80, 2893-2898, 1975.

Phillips, R.J., N.H. Sleep, and W.B. Banerdt, Permanent uplift in magmatic systems with application to the Tharsis region of Mars, $J$. Geophys. Res., 95, 5089-5100, 1990.

Plescia, J.B., Recent flood lavas in the Elysium region of Mars, karus, $88,465-490,1990$

Plescia, J.B., and R.S. Saunders, The chronology of the Martian volcanoes, Proc. Lunar Planet. Sci. Conf.. IOth, 2841-2859, 1979.

Richards, M.A., and B.H. Hager, Geoid anomalies in a dynamic Earth, J. Geophys. Res., 89, 5987-6002, 1984

Schubert, G. and T. Spohn, Thermal history of Mars and the sulfur content of its core, J. Gesphys. Res., 95, 14,095-14,104, 1990.

Schubert, G., D. Bercovici, and G.A. Glatzmaier, Mantle dynamics in Mars and Venus: Influence of an immobile lithosphere on threedimensional mantle convection, J. Geophys. Res.. 95, 14,105$14,129,1990$.

Schultz, R.A., and M.T. Zuber, Observations, models, and mechanisıns of failure of surface rocks surrounding planetary surface loads, J. Geophys. Res., 99, 14,691-14,702, 1994.

Scott, D.H., and K.L. Tanaka, Mars Tharsis region: Volcanotectonic events in the stratigraphic record, Proc. Lunar Planet. Sci. Conff., 11th, 2403-2421, 1980.

Sjogren, W.L., Mars gravity: High-resolution results from Viking Orbiter 2, Science. 203, 1006-1010, 1979.

Sjogren, W.L., and S.J. Ritke, Mars: Gravity data analysis of the Crater Antoniadi, Geophys. Res. Lett., 9. 739-742, 1982.

Sjogren, W.L., and R.N. Wimberley, Mars: Hellas Planitia gravity analysis, lcarus, 45, 331-338, 1981.

Sleep, N.H., and R.J. Phillips, An isostatic model for the Tharsis province, Mars, Geophys. Res. Lett., 6. 803-806, 1979.

Sleep, N.H., and R.J. Phillips, Gravity and lithospheric stress on the terrestrial planets with reference to the Tharsis region of Mars, $J$. Geophys. Res., 90, 4469-4489, 1985

Smith, D.E., and M.T. Zuber, Progress in improving our knowledge of the shape of Mars from occultations (abstract), Lunar Planet. Sci., 26. 1315-1316, 1995

Smith, D.E., F.J. Lerch, J.C. Chan, D.S. Chinn, H.B. Iz, A. Mallama, and G.B. Patel, Mars gravity field error analysis from simulated radio tracking of Mars Observer, J. Geophys Res., 95, 14,155$14,167,1990$.

Smith, D.E., F.J. Lerch, R.S. Nerem, M.T. Zuber, G.B. Patel, S.K. Fricke, and F.G. Lemoine, An improved gravity model for Mars: Goddard Mars Model 1, J. Geophys. Res., 98, 20,871-20,889, 1993.

Solomon, S.C., and J.W. Head, Evolution of the Tharsis province of Mars: The importance of heterogeneous lithospheric thickness 
and volcanic construction, J. Geophys. Res., 87, 9755-9774, 1982.

Solomon, S.C., R.C. Comer, and J.W. Head. The evolution of impact basins: Viscous relaxation of topographic relief, $J$. Geophys. Res., 87, 3975-3992, 1982.

Solomon, S.C., W.L. Sjogren, and S.R. Bratt, The structure of the Isidis Basin, Mars, from gravity anomalies (abstract), Lunar Planet. Sci. 14, 725-726, 1983.

Solomon, S.C., D.L. Anderson, W.B. Banerdt, R.G. Butler, P.M. Davis, F.K. Duennebier, Y. Nakamura, E.A. Okal, and R.J. Phillips, Scientific rationale and requirements for a global seismic network on Mars, LPI Tech. Report 91-02, 51 pp., 1991.

Stein, C.A., and S. Stein, A model for the global variation in oceanic depth and heat flow with lithospheric age. Nature, 359, 123129, 1992.

Tanaka, K.L., and D.H. Scott, Eruptive history of the Elysium volcanic province of Mars (abstract), Lunar Planes. Sci., 18, 998-999, 1987.

Turtle, E.P., and H.J. Melosh, Martian elastic lithospheric thickness from flexural and gravitational modeling (abstract), Lunar Planet. Sci., 26, 1427-1428, 1995.

U.S. Geological Survey, Topographic maps of the western, eastern equatorial and polar regions of Mars, Misc. Inv. Map 1-2030, 1989.
Willemann, R.J., and D.L. Turcotte, The role of lithospheric stress in the support of the Tharsis Rise, J. Geophys. Res., 87, 9793. $9801,1982$.

Zuber, M.T., D.E. Smith, S.C. Solomon, D.O. Muhleman, J.W. Head, J.B. Garvin, J.B. Abshire, and J.L. Bufton. The Mars Observer Laser Altimeter investigation, J. Geophys. Res., 97, 7781-7797. 1992.

Zuber, M.T., B.G. Bills, H.V. Frey, W.S. Kiefer, R.S. Nerem, and J.H. Roark, Possible hexural signatures around Olympus and As. craeus Montes, Mars (abstract), Lunar Planet. Sci., 24, 1591 1592, 1993.

B.G. Bills, Geodynamics Branch, Code 921, Goddard Space Flight Center, Greenbelt, MD 20771.

W.S. Kiefer, Lunar and Planetary Institute, 3600 Bay Area Blyd., Houston. TX 77058 (e-mail: kiefer@lpi3.jsc.nasa.gov).

R.S. Nerem, Dept. of Acrospace Engineering and Engineering Mechanics, University of Texas, Austin. TX 78712.

(Received July 6, 1994; revised September 27. 1995; accepted December 2, 1995). 